Running head: Autonomous wildland fire sentry

\title{
Autonomous field-deployable wildland fire sensors
}

\author{
R. Kremens ${ }^{A D}$, J. Faulring ${ }^{B}$, A. Gallagher ${ }^{C}$, A. Seema ${ }^{B}$, A. Vodacek $^{A}$ \\ ${ }^{A}$ Rochester Institute of Technology, Center for Imaging Science, 54 Lomb Memorial \\ Drive, Rochester NY 14623, USA \\ ${ }^{\mathrm{B}}$ Rochester Institute of Technology, Department of Computer Engineering, 83 Lomb \\ Memorial Drive, Rochester NY 14623, USA \\ ${ }^{\mathrm{C}}$ Rochester Institute of Technology, Department of Electrical Engineering, 79 Lomb \\ Memorial Drive, Rochester NY 14623, USA \\ ${ }^{\mathrm{D}}$ Corresponding author; Telephone: +1 585475 7286; FAX: +1 585475 5988; e-mail \\ $\underline{\text { rkremens@cis.rit.edu }}$
}

Abstract. An Autonomous Fire Detector (AFD) is a miniature electronic package combining position location capability (using the Global Positioning System [GPS]), communications (packet or voice-synthesized radio), and fire detection capability (thermal, gas, smoke detector) into an inexpensive, deployable package. The AFD can report fire-related parameters, like temperature, carbon monoxide concentration, or smoke levels via a radio link to firefighters located on the ground. These systems are designed to be inserted into the fire by spotter planes at a fire site or positioned by firefighters already on the ground. AFDs can also be used as early warning devices near critical assets in the urban-wildland interface. AFDs can now be made with commercial off-the-shelf components. Using modern micro-electronics, an AFD can 
operate for the duration of even the longest fire (weeks) using a simple dry battery pack, and can be designed to have a transmitting range of up to several kilometers with current low power radio communication technology. A receiver to capture the data stream from the AFD can be made as light, inexpensive and portable as the AFD itself. Inexpensive portable repeaters can be used to extend the range of the AFD and to coordinate many probes into an autonomous fire monitoring network.

Keywords: Fire monitoring; field systems; fire detection; local fire detection; remote sensing.

\section{Introduction}

One of the major problems in understanding and combating wildland fires is monitoring the time history of the fire (Chandler 1983). Understanding the size, location, and speed of advance of the fire front is critical to optimal allocation of fire fighting resources and to maintaining safety of the fire crew. Investigation of major wildland fire accidents involving loss of life often shows that the crews became imperiled because of insufficient or untimely information about the location and speed of advance of the fire (Rothermel 1993).

An autonomous fire detector (AFD) is a field deployed fire alarm that has the ability to remotely report its location and the presence of a fire in the vicinity. A wildland fire can be detected by one or more inexpensive sensors in the AFD that detect smoke, carbon monoxide, methyl chloride, rapid temperature increases, or any of a number of other physical phenomena related to the presence of a wildland fire. The use of multiple sensors reduces the likelihood of false alarms without unduly complicating the device. The AFD may also be equipped to record 
and transmit other data affecting fire spread such as relative humidity and wind speed. The data gathered by the AFD can be transmitted in several ways: it can be recorded locally to get a postfire time history (if the AFD is recovered); or transmitted by radio in real time to individual firefighters equipped with appropriate receivers; or transmitted to a central receiver at the fire command center. In addition the AFD can also be used as an independent data collection device on prescribed fires, and when equipped with appropriate sensors, will assess such parameters as thermal output, temperature, gaseous product evolution and local weather data.

At present, once firefighters are on the ground near the fire site, they may be effectively blind to the activity of the fire. Spotter planes and other aircraft may periodically over fly the area and report the movement and location of the fire to the incident commander (IC), but often even this rudimentary data is lacking. The loiter time of aircraft over a fire is limited by fuel and cost considerations, and dissemination of data from the IC or aircraft control to crew units may be spotty and irregular. Data that is locally available to individuals or small fire fighting units on a continuous basis can vastly improve fire fighting efficiency and safety.

Much effort has been expended in modeling the movement of fires in wildland settings (Andrews 1986, Andrews and Bevins 1998, Finney 1998) but these models are only as good as the detailed weather, terrain, and fuel load information. Lacking precise information of the fire site, these complex fire models can predict fire behavior for short time periods, but must then be 'tuned' with actual data to obtain long-term accuracy. The fire models are similar to modern weather simulations that are periodically adjusted with measured weather data to provide accurate long-term modeling. Again, the availability of timely data over the entire fire area would increase the accuracy of model predictions and aid firefighters. 
Using AFDs and armed with handheld computers running these fire models, firefighters will

70 have accurate real-time data for model 'tuning', and may be able to more accurately predict fire

71 behavior based on past fire movement even when only very imprecise weather, fuel, and terrain

72 information is initially available. The ability to predict the movement of the fire is a powerful

73 advantage to fire logistics and firefighter safety.

74 The use of satellites to obtain fire data for model tuning is possible, but there are

75

76

77

78

79

80

81

82

83

84

85

86

87

88

89

90

91 complications imposed by limited satellite spatial resolution, complicated ground link equipment, and short satellite loiter time (for low Earth orbit satellites) over the target area. Real time data can be obtained using unmanned or remotely controlled unmanned flying vehicles (UFVs) flying over the fire site, but this solution is both complex and difficult to support in the field and requires additional worker training (and new infrastructure) to operate and maintain the UFV fleet. A small number of AFDs that are located in the forest provides fire spread data at low cost and with little additional effort in training or support. Following we present three AFD concepts that have different applicability and capabilities and show a prototype that was developed in our laboratory in a matter of only a few months.

\section{Operational considerations}

In use, the AFDs can be dropped from a spotter plane or manually positioned by fire crews over an area where a fire has previously been detected. The mechanical package of the AFD can be designed to be canopy penetrating (to descend to the forest floor) or canopy snagging (to hang in the upper branches of the canopy). The devices periodically report their position and fire status to each other, a central receiver, or to a radio receiving set provided to firefighters. 
After they are deposited in the fire area, the AFDs find their location (via their internal GPS

93 receiver) and report their initial position and fire alarm status via a radio link. Communication is 94 provided via a radio transceiver, which make AFD-to-AFD as well as AFD-to-base unit 95 communication possible. Communication range can be extended by providing message store

96

97

98

99

100

101

102

103

104

105

106 and forward or repeat capability on the AFD. A typical AFD message might then be as follows:

AFD unit identifier number, latitude, longitude, altitude, date, time, alarm status

1, alarm status 2...alarm status $N$.

This simple message can be transmitted digitally using any of a number of low-bandwidth encoding schemes (5 or 7 level radio-teletype), or can be transmitted via a synthesized voice.

Other more advanced network schemes are also possible, although these increase the complexity of both the software and hardware over the simple systems described here. Each AFD is programmed to transmit in sequence at a slightly different time in order to avoid AFD message collisions and interference (time division multiplexing [TDM]), even when operating on a single radio frequency (Figure 1). TDM is especially easy because the GPS provides an extremely accurate clock for synchronization of transmission times. 
AFD N

\section{AFD \#3}

AFD \#2

AFD \#1

GPS Synch

Pulse

Data Burst

Data Burst

Data Burst

Data Burst

107

108

109

110 the GPS.

111

112

113

114 A simple mode of operation of the AFD system is depicted in Figure 2. In this mode, the AFDs

115 operate independently of each other without a central control transceiver and report simple

116 synthesized voice messages to any firefighters within radio range on the ground. This message

117 contains the ID number of the AFD, its GPS position (latitude, longitude, and altitude), and the

118 alarm state of the device. The firefighter, equipped with nothing more than the present

119 VHF/UHF FM radio transceiver ('handi-talki') provided to US wildland firefighters, and with no

120 additional infrastructure, is able to receive voice AFD status on one of the unused radio

121 communication channels. This mode of operation is most suitable when a few ( 10) AFDs are

122 used on geographically small fires. 


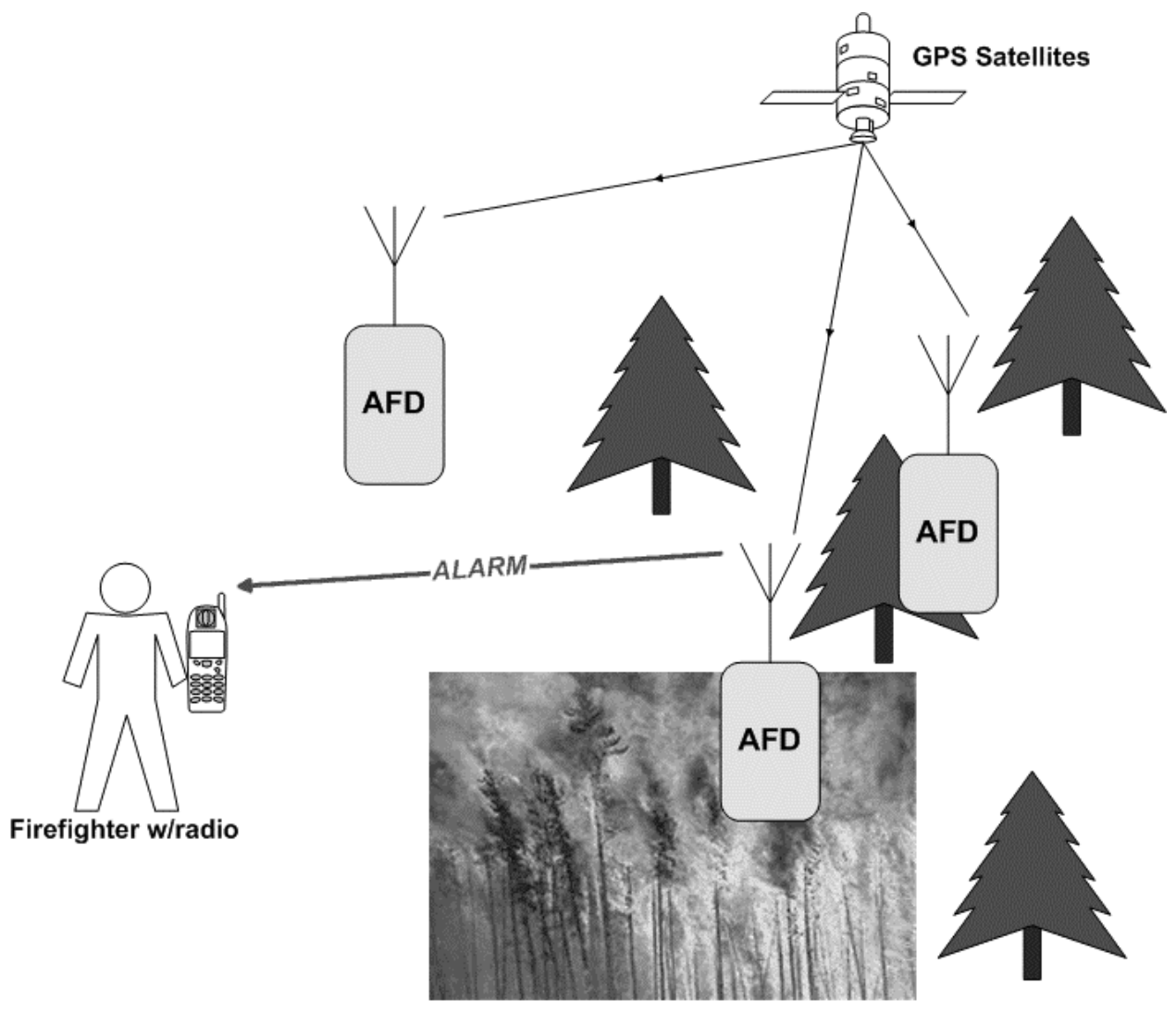

Fig. 2. Simple direct voice point-to-point AFD operation. The AFDs report via synthesized voice messages directly to radio receivers at the fire site.

\section{Mode 2: Networked operation}

Another option for AFD communication uses a digital data link with a network protocol. This

131 operational mode represents a relatively complex implementation of the AFD. A diagram of the communication links between the various units of a digital AFD system is shown in Figure 3. The AFDs will periodically report their status to each other and possibly also to a central control

134 transceiver unit. Since in the AFD system, messages are simple, short and repeated until the 135 alarm has passed, even if some message packets are lost, the alarm will still be received in a 
136 timely fashion. The individual messages have low information content and the required update

137 rate is very low (several times per minute), which adds to simplicity and reliability of the AFD

138 system.

139 On detection of a fire, the reporting AFD or AFDs will transmit an alarm to other AFDs in the

140 area and to the central transceiver. In this way the range of a single AFD can be extended

141 beyond the normal line-of-sight range of a single unit. Crews in the area can be alerted either

142 directly from the reporting AFD, or through alarm messages that are relayed from the control

143 transceiver. The control transceiver can overlay geographical information system (GIS) maps

144 and data with the location and alarm state of the AFD, and can present this data to the incident

145 commander or other personnel at the fire command post. Upon passage of the fire front, the

146 AFDs may be recovered using an audible and/or radio homing signal. 


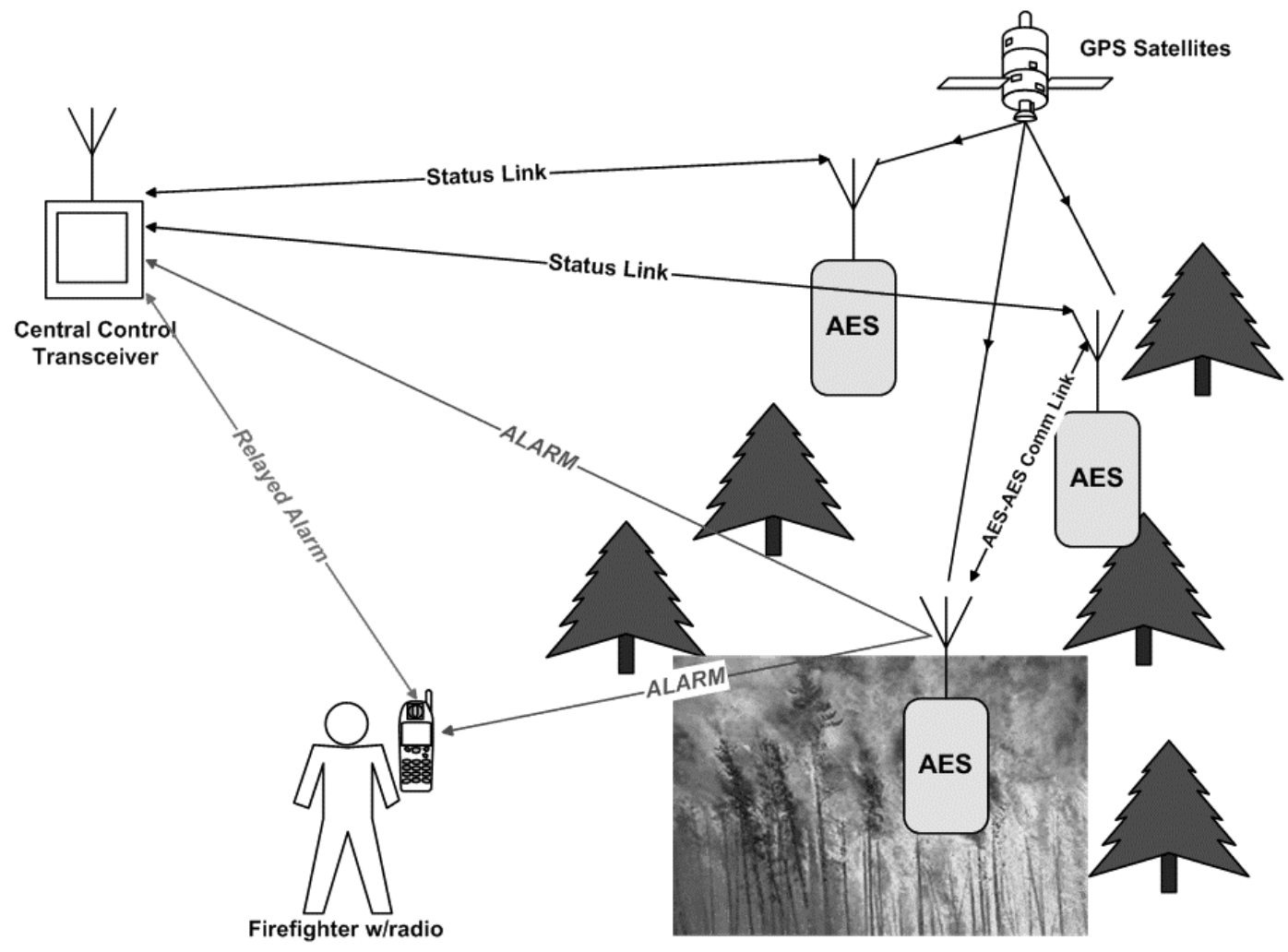

Fig. 3. Deployment and communication between AFDs and base units, other AFDs and firefighters in a fully networked system.

Point-to-point AFDs can be installed before a prescribed burn to both increase safety of the fire team and provide valuable measurements of temperature (air, ground and/or bole), humidity, wind velocity, or other parameters that are related to fire phenomena or fire ecology. Since a number of these AFDs can be deployed at a burn, a time history field of the measured parameter may be conveniently obtained and relayed to scientists or fire managers during the burn operation. The resulting data field may be analyzed later or used as input to models. For example, burn scar ground temperature as a function of time after the passage of the flaming 
161 front is poorly known. This data is necessary to determine how long a fire would remain visible 162 to a remote sensing platform using infrared heat-detecting sensors after passage of the flaming

163 fire front. The AFD system is ideally suited to this position and time sensitive data-gathering task. A diagram of this AFD implementation is shown in Figure 4.

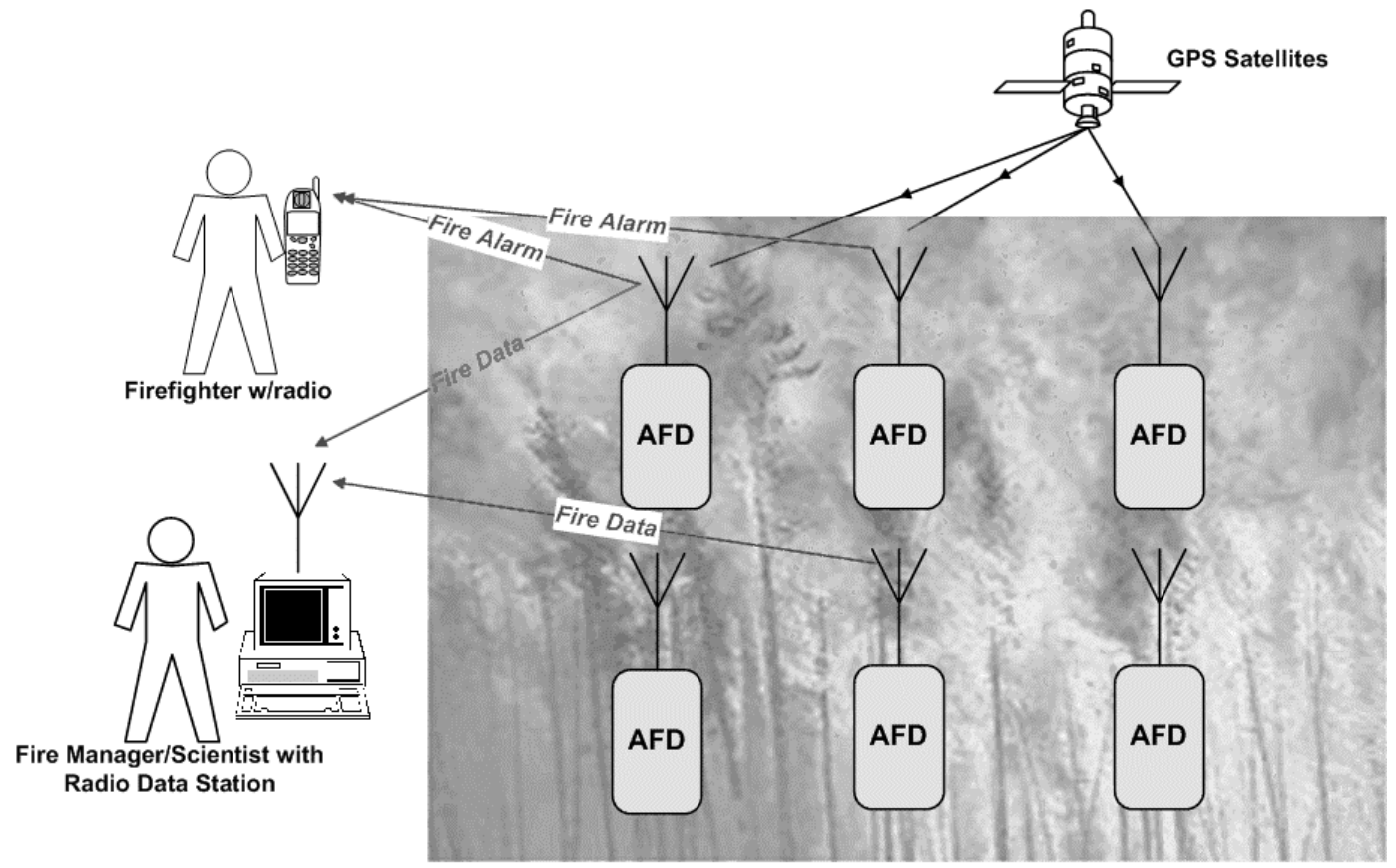

Field of AFDs in Prescribed Burn Study Area

Fig. 4. Use of the AFD during a prescribed burn to collect data and increase worker safety. A field of AFDs is employed that both record data locally for later retrieval and forward fire passage information for safety purposes.

\section{AFD Design Concept}

A block diagram of the AFD is shown in Figure 5. A microprocessor coordinates inputs from

174 the global positioning system receiver and the fire sensors and generates the communication and

175 modulation stream for the radio transceiver system. This communication stream can be digital, 
employing packet or radio-teletype encoding technology, or can be a synthesized voice, as discussed above. A multi-channel analog-to-digital converter processes the analog information from several fire and environment sensors and produces a digital data stream that is analyzed by the supervisory microprocessor.

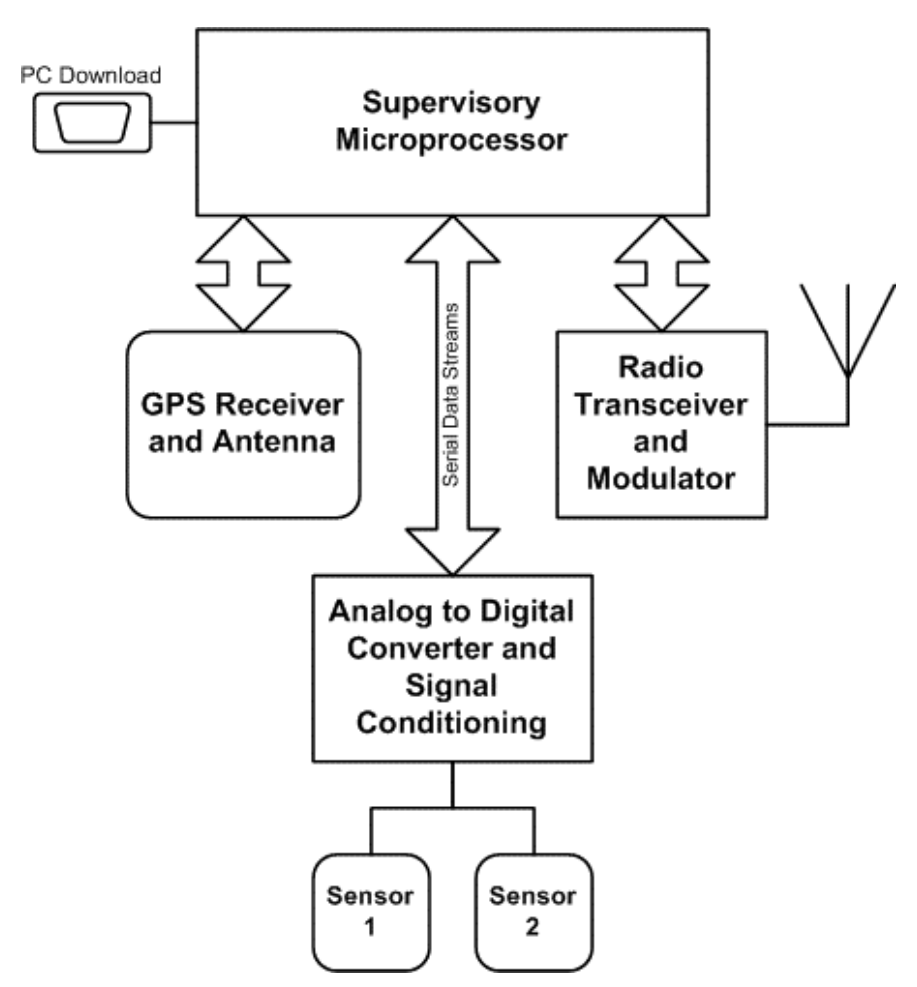

Fig. 5. Functional blocks of the AFD. A microprocessor coordinates inputs from the GPS and sensors to produce a digital data stream that is transmitted by radio. Only two sensors are shown, but more can be added to reduce false alarms and improve detection capability.

Several fire sensors may be used in an AFD. These sensors could be smoke detectors (photoelectric or ionization), gas detectors (combustion precursor gases, carbon dioxide, carbon monoxide, etc.), thermal (temperature), passive microwave or optical radiation (visible or infrared) detectors. In general, each detector requires a different analog signal processing 'front end', which is included with the detector on an interchangeable module that plugs into the main 
AFD 'motherboard'. The AFD motherboard performs analog-to-digital conversion on the

192 conditioned signal from the sensors and determines whether or not to issue an alarm. The alarms

193

194

195

196

197

198

199

200

201

202

203

204

205

206

207

208

209

210

211

212

213 can be based on change (e.g. temperature, humidity) or level ( $\mathrm{CO}$ concentration). The use of more than one inexpensive detector can greatly reduce the probability of false alarms while not significantly increasing the cost. We are currently executing a test program to evaluate and optimize several fire sensor configurations during controlled wildland fires.

The AFD will be programmed to observe and report sensor input periodically and to 'sleep' in the intervening periods to conserve battery energy. The prototype AFD has the ability to switch off power to a number of external devices during these 'sleep' periods, further reducing power requirements and extending battery life.

\section{Prototype Design}

We have constructed a flexible experimental prototype AFD that conforms to the basic design discussed above. Key prerequisites of the design are cost effectiveness, durability, low power consumption and adequate transmitting range. Any design must be sensitive to the multiple design constraints of low cost, ruggedness, low power consumption, and adequate transmitting range. No particular effort was made to miniaturize or reduce the cost of the prototype, since it was envisioned that these early devices should be flexible and easy to modify for development and optimization. 'Production' AFDs may be significantly smaller, simpler, and less expensive than the prototype described here.

The prototype uses commercially available components and systems where possible. The custom prototype printed wiring board has the components necessary to transmit and receive 
214 digital messages, transmit audio messages, and digitize up to 9 analog sensor signals. For 215 communication we use frequency modulated (FM) radio transceivers operating in the US 216 Amateur Radio Service portion of the VHF band (145 MHz). The AFD can transmit information 217 using either audio frequency shift keying (AFSK) for digital information or a voice synthesizer 218 to transmit audio alerts. A radio receiver-demodulator attached via a serial link to a laptop 219 computer receives the digital data stream and displays the messages, while the voice 220 transmissions are heard on the speaker of any radio capable of receiving on the $145 \mathrm{MHz} \mathrm{VHF}$ 221 band. All of the components, including the radio transceivers, were obtained commercially. A 222 detailed block diagram of the AFD describing the electronic components is shown in Figure 6 223 and a photograph of the completed printed circuit board is shown in Figure 7.

224 In order to speed development, a Parallax, Inc. Basic Stamp 2 microcomputer module (BS2E) 225 was used for the central processing unit. The BS2 has 16 digital input/output lines that can be 226 programmed individually to perform a number of functions. This microcomputer module has 227 been optimized for control applications and is programmed with the powerful PBASIC 228 programming language. Programs are developed on a PC-compatible computer and downloaded 229 to the BS2, where they reside on an electrically erasable programmable memory (EEPROM). 230 The combination of powerful input/output based programming language and self-contained 231 development system makes the BS2 very easy to use for rapid development of simple 232 applications. Detailed information about this processor can be found at the company's web site $233[5]$. 


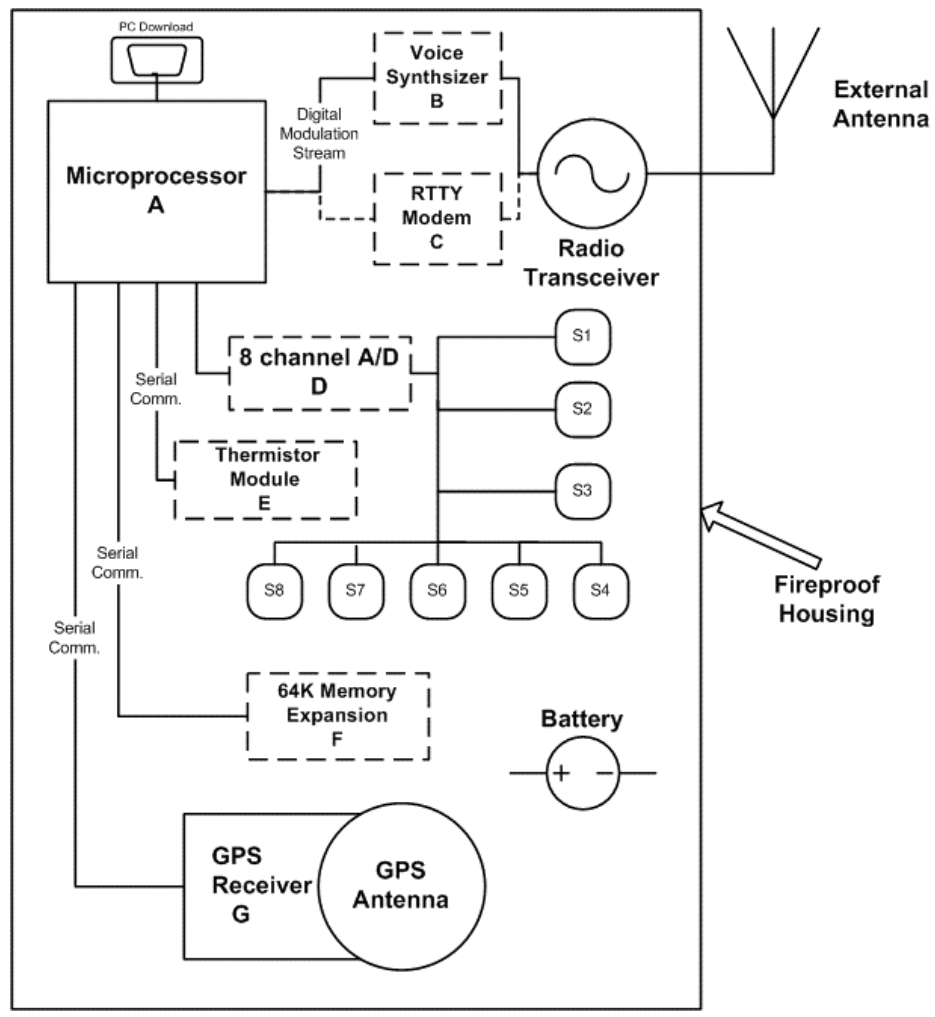

236 Fig. 6. Block diagram of the AFD, showing the logical and signal interconnections between the fire sensors, global

237 positioning system receiver, radio transceiver and power system. Components: S1 - S8 - Fire sensors; A Central 238 control microprocessor; B - Voice synthesizer components; C - Radioteletype MODEM; D - Analog-to-digital 239 converter; E - Precision thermistor interface; F - Serial non- volatile memory; G - GPS receiver module. 


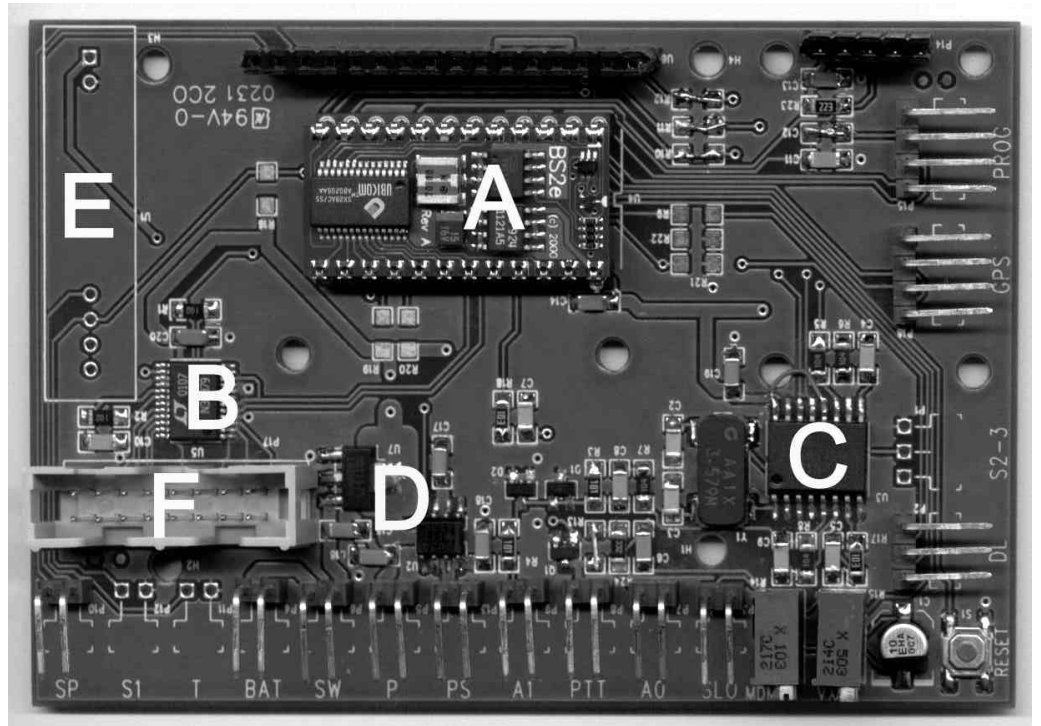

243 Fig. 7. Photograph of the prototype AFD. Connections to the system are made via jacks on the periphery of the 244 circuit board. The AFD is shown in the digital link communication configuration. The speech synthesizer is 245 plugged into the socket on the top of the board near the microprocessor. A - Parallax Basic Stamp microprocessor; B 246 - Linear Technology LTC1598 8-channel, 12-bit analog-to-digital converter; C - MX-Com MX 614 Modem IC; D 247 Power management circuitry; E - YSI 4800 LC precision thermistor module (not installed) ; F - 8-channel analog 248 input connector.

The prototype AFD has eight 12-bit analog input channels to accept input from fire sensors. A Linear Technology, Inc. LTC1598 ADC communicates with the microprocessor via a 3-wire 252 synchronous serial link. We are currently evaluating several sensor types for suitability in this application. We have successfully detected test fires using both commercial ionization chamber

254 smoke detector and carbon monoxide detector modules and by measuring a rapid rise in ambient temperature using thermistors.

256 In addition to the analog inputs, several digital input/output ports are available. These ports 257 can be used to select among several operation modes, trigger a self-test, activate the device after 258 deployment, or to drive light emitting diodes (LED) to provide visual feedback during operation. 
An audio alert can also be included to provide local indication of an alarm condition and an audio homing signal to aid in recovery of the device from the field.

Asynchronous serial communication in IEEE RS-232 format is used to communicate between the AFD and GPS unit. A commercial GPS receiver (Garmin Model GPS-35) sends ASCII information indicating time, latitude and longitude over one serial link to the microprocessor in National Marine Electronics Association NMEA-0183 standard format. A precision one-second timing pulse is also generated by the GPS receiver. This timing pulse is used to synchronize TDM transmissions. transmission of an ASCII digital data stream at 1200 bits per second. The other modulator is a

271 voice synthesizer manufactured by Quradravox, Inc. ASCII commands to this synthesizer generate natural sounding voice messages. The audio output from either of these modulators drives a commercial 5-watt VHF-FM transceiver. Each unit transmits its alarm status at a predetermined unique time delay after some 'zero' time (say, the start of a minute). With a relatively small antenna, this transmitter has a worst case range of more than $5 \mathrm{~km}$, depending on the terrain between the AFD and the receiving station.

77 A flow diagram of the software written for the prototype AFD is shown in Figure 8. The 278 powerful control-oriented PBASIC language simplified programming and reduced the initial 279 effort to just a few weeks. There are other branch points in the software flow (not shown in 280 Figure 6) that provide test functions (such as sensor and battery test) and readiness verification. 281 Another Basic Stamp BS2 has been programmed as an input test set. This device produces 
282 simulated signals for the input switches and two analog voltages to represent sensor outputs and 283 allows us to test the AFD without directly stimulating the sensors.

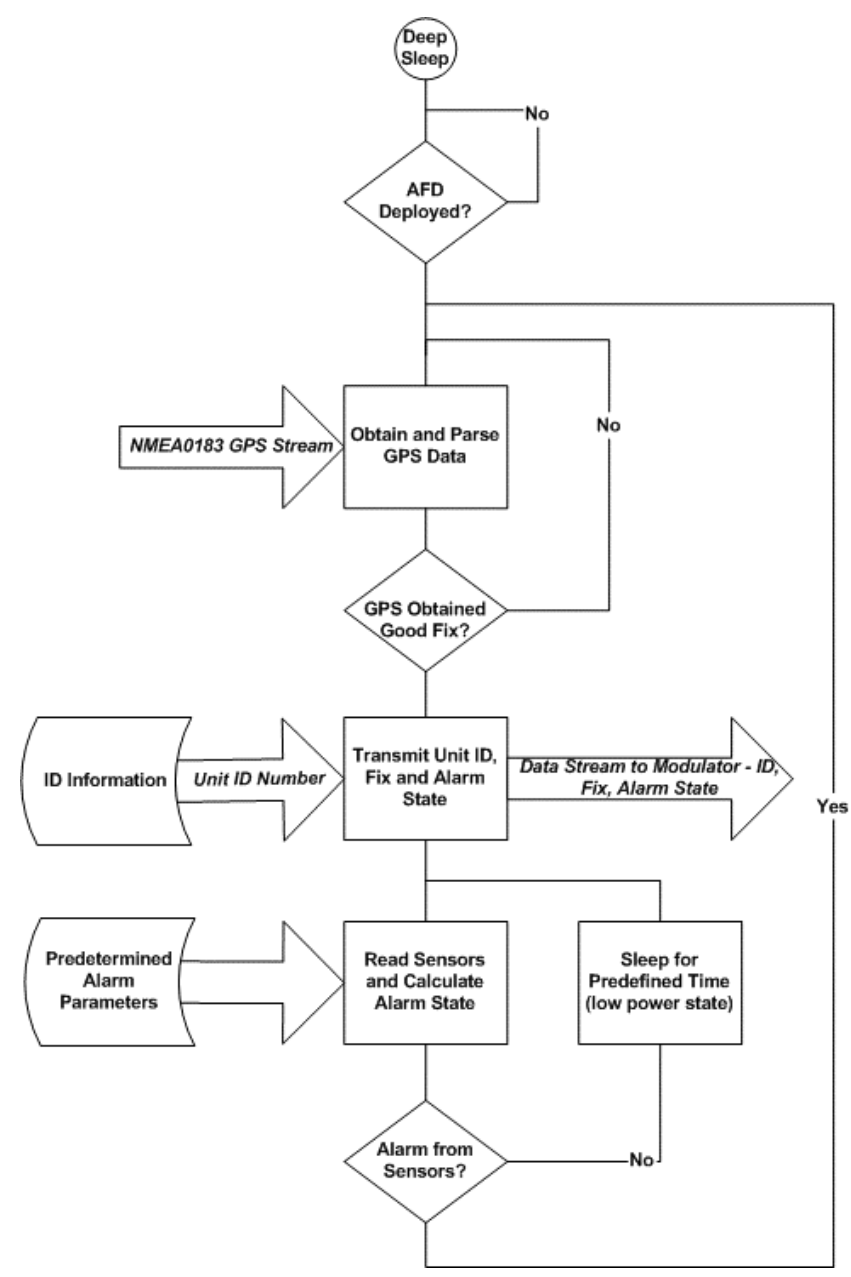

285 Fig. 8. Simplified software flow diagram for the AFD. Test routines and data communication (TDM timing and 286 message generation) are not shown.

Fire Sensors

290 The AFD as currently configured can use up to eight analog input sensors. An additional 291 precision temperature measurement channel is available for high-accuracy data acquisition 292 applications, but in general would not be required for alarm use. The AFD can measure 
temperature, $\mathrm{CO}, \mathrm{CO}_{2}$ and other combustion by-product gas concentrations, humidity, wind 294 speed, optical emissions and thermal flux simultaneously if necessary. Inexpensive sensors have 295 been developed by the structure-fire alarm community and can be adapted easily to this 296 application.

297 We have found that a fire detection sensor using two thermistors in a differential configuration 298 provides sensitive and inexpensive local detection of a fire. One thermistor is thermally 299 insulated and located in the interior of the AFD case. The thermal time constant of this sensor is 300 about 30 minutes. The other thermistor is mounted on the exterior. A rapid rise in the 301 temperature difference between the interior and exterior thermistor (time constant $<1$ minute) 302 indicates the presence of a nearby fire, since the interior thermistor cannot change temperature 303 rapidly due to its long thermal time constant. Normal daily temperature variations produce 304 roughly equal temperature changes in the interior and exterior thermistor. A diagram of this 305 sensor scheme is shown in Figure 9.

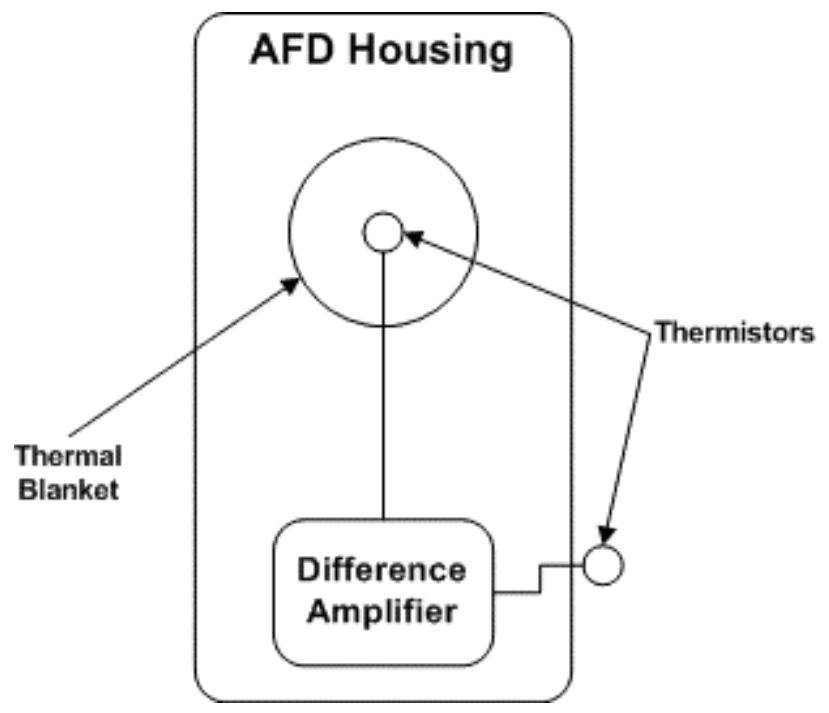




\section{Conclusion}

311

312 We have demonstrated the concept and electronics for an autonomous fire detector that may be

313 used as a fire sentry when fighting wildland fires. By keeping both the principles of operation 314 and physical hardware simple, the device has been prototyped rapidly. Several inexpensive 315 sensor designs have been identified. The same electronic package can also be used for safety and 316 fire monitoring during prescribed burns.

317 We are currently evaluating multi-sensor fire detector packages on small locally constructed 318 test fires. We will test a complete mechanical and electrical package with an extended range

319 VHF radio transmitter during prescribed fires in the Northwest Rocky Mountains during the Fall 320 of 2002.

\section{Acknowledgements}

The National Aeronautics and Space Administration supported this work under Grant NAG5-

325 10051. Its financial support has been greatly appreciated.

\section{References}

Andrews PL (1986) BEHAVE: fire behavior prediction and fuel modeling system - BURN

330 subsystem, Part I. United States Department of Agriculture, Forest Service, Intermountain

331 Research Station General Technical Report, INT-194, Ogden, Utah. 130 pages. 
333 Andrews PL, Bevins CD (1998) Update and expansion of the BEHAVE fire behavior prediction

334 system. $3^{\text {rd }}$ International Conference on Forest Fire Research; $14^{\text {th }}$ Conference on Fire and Forest

335 Meterology, 21 - 22 November 1998, Luco-Coimbra, Portugal, Vol. I, pp. 733-740.

336

337 Chandler C, Cheney P, Thomas P, Traubaud L, Williams D (1983) Fire in forestry, Vol. II, (John

338 Wiley and Sons: New York, USA)

339

340 Finney MA (1994) FARSITE: a fire area simulator for fire managers. The Biswell Symposium, 341 February 15-17, 1994. Walnut Creek, California.

342

343 Parallax Corporation (2001) http://parallaxinc.com

344

345 Rothermel RC (1993) Mann gulch fire: a race that couldn't be won. United States Department of 346 Agriculture, Forest Service, Intermountain Research Station General Technical Report, INT-299, 347 Ogden, Utah. 10 pages. 